PREPARED FOR THE U.S. DEPARTMENT OF ENERGY, UNDER CONTRACT DE-AC02-76CH03073

PPPL-3583

PPPL-3583

UC-70

\title{
Measurement of Lower-Hybrid Drift Turbulence in a Reconnecting Current Sheet
}

T.A. Carter, H.Ji, F. Trintchouk, M. Yamada, R.M. Kulsrud

June 2001
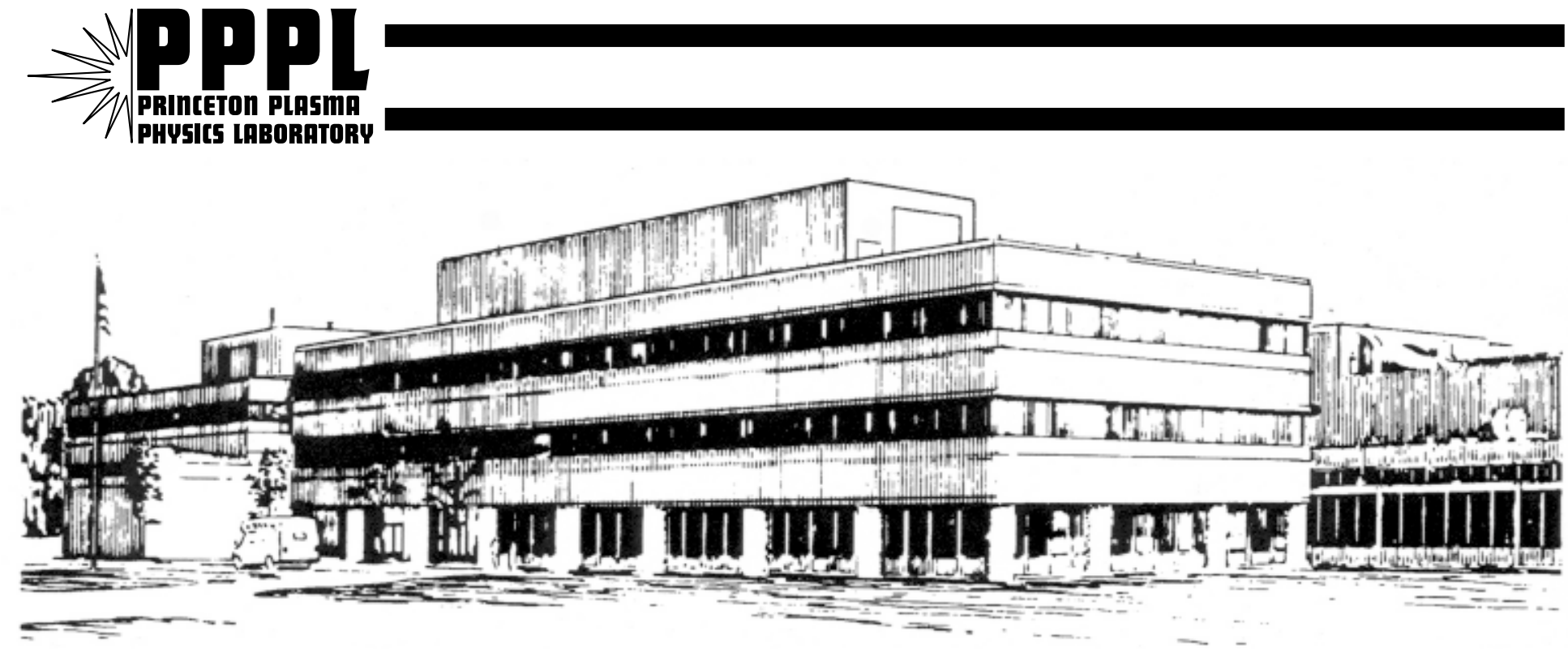

PRINCETON PLASMA PHYSICS LABORATORY PRINCETON UNIVERSITY, PRINCETON, NEW JERSEY 


\section{PPPL Reports Disclaimer}

This report was prepared as an account of work sponsored by an agency of the United States Government. Neither the United States Government nor any agency thereof, nor any of their employees, makes any warranty, express or implied, or assumes any legal liability or responsibility for the accuracy, completeness, or usefulness of any information, apparatus, product, or process disclosed, or represents that its use would not infringe privately owned rights. Reference herein to any specific commercial product, process, or service by trade name, trademark, manufacturer, or otherwise, does not necessarily constitute or imply its endorsement, recommendation, or favoring by the United States Government or any agency thereof. The views and opinions of authors expressed herein do not necessarily state or reflect those of the United States Government or any agency thereof.

\section{Availability}

This report is posted on the U.S. Department of Energy's Princeton Plasma Physics Laboratory Publications and Reports web site in Calendar Year 2001. The home page for PPPL Reports and Publications is: http://www.pppl.gov/pub_report/

DOE and DOE Contractors can obtain copies of this report from:

U.S. Department of Energy

Office of Scientific and Technical Information

DOE Technical Information Services (DTIS)

P.O. Box 62

Oak Ridge, TN 37831

Telephone: (865) 576-8401

Fax: (865) 576-5728

Email: reports@adonis.osti.gov

This report is available to the general public from:

National Technical Information Service

U.S. Department of Commerce

5285 Port Royal Road

Springfield, VA 22161

Telephone: 1-800-553-6847 or

(703) 605-6000

Fax: (703) 321-8547

Internet: http://www.ntis.gov/ordering.htm 


\title{
Measurement of lower-hybrid drift turbulence in a reconnecting current sheet
}

\author{
T.A. Carter, H. Ji, F. Trintchouk, M. Yamada, R.M. Kulsrud \\ Princeton University, Plasma Physics Laboratory, P. O. Box 451, Princeton, NJ, 08543
}

\begin{abstract}
We present a detailed study of fluctuations in a laboratory current sheet undergoing magnetic reconnection. The measurements reveal the presence of lower hybrid frequency range fluctuations on the edge of current sheets produced in the Magnetic Reconnection Experiment (MRX). The measured fluctuation characteristics are consistent with theoretical predictions for the lower-hybrid drift instability (LHDI). Our observations suggest that the LHDI does not provide any significant turbulent resistivity in MRX current sheets. PACS numbers: 52.35.Vd, 52.35.Qz, 52.35.Ra, 52.72.+v
\end{abstract}

Typeset using REVTEX 
Magnetic reconnection [1] is an important process in magnetized plasmas in which a rapid release of magnetic energy and changes in magnetic topology can occur even in highly conducting plasma. Recent experiments [2] and simulations [3] have shown that current sheets associated with reconnection have widths on the order of the ion skin depth $\left(c / \omega_{\mathrm{p}, \mathrm{i}}\right)$, a scale on which the magnetohydrodynamic (MHD) model is likely to be inaccurate. There are two theoretical models in the literature which address the problem of fast reconnection in non-MHD current sheets. The first invokes microinstability-produced anomalous resistivity [4] which enhances the rate of magnetic field dissipation and broadens the current layer to assist in the outflow of mass. The second theoretical model invokes non-dissipative terms in the generalized Ohm's law, specifically the Hall term, which can lead to laminar fast reconnection in collisionless plasmas [5]. Experimental data on the nature and role of turbulence in reconnecting current sheets is needed to resolve the obvious controversy presented by these two theoretical perspectives. Motivated by this goal, we have carried out a detailed experimental study of fluctuations which has lead to the first observation of the lower-hybrid drift instability in a laboratory current sheet.

The lower-hybrid drift instability (LHDI) [6] should be driven by cross-field currents and density gradients present in current sheets. It has a real frequency near the lower hybrid frequency $\left(\omega_{\mathrm{LH}} \sim \sqrt{\Omega_{\mathrm{e}} \Omega_{\mathrm{i}}}\right)$ and inverse wavenumber near the electron gyroradius $\left(k \rho_{\mathrm{e}} \sim 1\right)$. The LHDI has been proposed as a source of anomalous resistivity in reconnecting current sheets [7]. However, theoretical studies of the LHDI have revealed that the instability is linearly stabilized by large plasma beta [6], which should confine the instability to the edges of a current sheet. Three-dimensional particle simulations [8] and Hall MHD simulations [9] have actually shown that the LHDI does develop in the current sheet, but have conflicting views on the role of the turbulence: the former shows that LHDI can assist in driving reconnection (through profile modification or by assisting in electric field penetration), while the latter shows that LHDI can actually slow reconnection relative to laminar two-dimensional simulations. There is observational evidence for the existence of the LHDI in the magnetotail current sheet [10], where it may trigger current disruptions [11]. Earlier laboratory studies 
of fluctuations have revealed evidence for unstable ion acoustic and whistler waves in current sheets [12], but the ion gyroradius in these experiments was larger than the apparatus size and excitation of instabilities such as the LHDI might not have been possible.

The experiments reported here were performed in the Magnetic Reconnection Experiment (MRX) [13]. In MRX well diagnosed, long lived current sheets $\left(\tau \gg \tau_{\mathrm{A}}\right)$ are formed in plasmas in which MHD is satisfied in the bulk of the plasma $\left(S \gg 1, \rho_{\mathrm{i}} \ll L\right.$ away from the current sheet). A schematic of the MRX device is shown in Fig. 1, along with a single magnetic field line indicating the current sheet location. Collisionality in MRX current sheets is characterized by the parameter $\lambda_{\operatorname{mfp}} / \delta$, where $\delta$ is the width of the current sheet and $\lambda_{\mathrm{mfp}}$ is the electron mean free path. Two observations, which motivate the study of turbulence, have been made as the collisionality was lowered $\left(\lambda_{\operatorname{mfp}} / \delta\right.$ is increased $)$ in MRX. The first is that the measured toroidal reconnection electric field, $E_{\theta}$, is no longer balanced by classical collisional drag at the center of the current sheet, $E_{\theta} / \eta_{\mathrm{sp}} j_{\theta} \gg 1$, where $\eta_{\mathrm{sp}}$ is the classical Spitzer perpendicular resistivity [14]. The second is that direct, nonclassical ion heating has been observed during reconnection in MRX and the heating was found to strengthen with lower collisionality [15]. One possible explanation for these observations is the presence of turbulence in low-collisionality MRX current sheets, which creates a turbulent anomalous resistivity $\eta^{*}>\eta_{\mathrm{sp}}$ (so that $E_{\theta} / \eta^{*} j_{\theta}=1$ ) and directly heats the ions. Detailed high-frequency fluctuation measurements were made in MRX in order to test this hypothesis. Two types of diagnostics were used for this study, floating double Langmuir probes with 0.1 to $0.7 \mathrm{~mm}$ diameter tungsten tips and magnetic pickup loops (3 $\mathrm{mm}$ diameter, 80 turns). In both types of probes, miniature buffer amplifiers were embedded near the probe tips for improved immunity from firing circuit noise and for ease of impedance matching. The fluctuation probes used in these studies were inserted into the MRX current sheet radially, as shown in Fig. 1(a). Radial profiles were obtained through shot-to-shot positioning of the probes and through averaging over several shots at each position. The experiments reported here were performed in current sheets where no toroidal guide field was imposed.

Typical time traces of the total plasma current and measured signal from a floating dou- 
ble Langmuir probe located at the inner edge of the current sheet $(R=0.34 \mathrm{~m})$ are shown in Fig. 1(b). High frequency signals are seen to arise with the formation of the current sheet and persist for tens of microseconds. The amplitude of the fluctuations when normalized to the measured electron temperature $\left(e \delta \phi_{\mathrm{f}} / T_{\mathrm{e}}\right)$ is a few percent. A fast Fourier transform of this signal shows a rather broad power spectrum located near the lower hybrid frequency $\left(f_{\mathrm{LH}} \sim 16 \mathrm{MHz}\right.$, computed from the locally measured magnetic field). High frequency magnetic field fluctuations are also observed concomitantly in space and time with the floating potential signals, with very similar frequency spectrum. The amplitude of the measured magnetic fluctuations is on the order of $5-10 \mathrm{G}\left(\delta B / B_{\mathrm{o}}\right.$ a few percent $)$ and is strongest in the reconnecting field component $\left(B_{\mathrm{z}}\right)$.

The detailed dependence of the frequency spectrum of the fluctuations on the local lower hybrid frequency was explored through varying the capacitor bank voltage (which changes the peak field in the current sheet) and the mass of the working gas. Fig. 2(a) shows example floating potential fluctuation power spectra at different local field values and with two different fill gases, hydrogen and helium. There is an upward shift in the power spectrum with increasing field strength and decreasing ion mass, consistent with the shift in the local lower hybrid frequency. Fig. 2(b) shows the power spectrum of the fluctuations averaged over 97 discharges, including both hydrogen and helium shots, with the frequency normalized to the local lower hybrid frequency of each discharge. The averaged spectrum is quite broad, but is clearly situated near the lower hybrid frequency, consistent with theoretical expectations for the LHDI.

Fig. 3(a) shows average radial profiles of the fluctuating floating potential amplitude superimposed on the deduced average current density profile at four times during a set of more than 200 low-collisionality $\left(\lambda_{\mathrm{mfp}} / \delta \sim 5-10\right)$ MRX discharges. The magnetic field profile in MRX is well described by the Harris sheet equilibrium model [2], and the current profiles shown in this figure are computed through fitting the average magnetic field measurements to the Harris profile and then deriving the current density from the fit. The fluctuation amplitude is concentrated on the inner edge of the current sheet and is observed 
to grow as the current sheet forms and reconnection begins but then decay even while reconnection persists. In order to explain the spatial profiles and temporal behavior of the measured instability amplitude, linear calculations of the local growth rate of the LHDI in the MRX current sheet were performed. An electrostatic local theory was employed which includes finite plasma beta effects through $\nabla B$ drift corrections to the electron orbits. The following expression is found for the dispersion of the LHDI in this limit $[16,6]$ :

$$
\begin{gathered}
0=1-\frac{\omega_{\mathrm{p}, \mathrm{i}}^{2}}{2 k^{2} v_{\mathrm{th}, \mathrm{i}}^{2}} \mathrm{Z}^{\prime}\left(\frac{\omega-\mathbf{k} \cdot \mathbf{V}}{k v_{\mathrm{th}, \mathrm{i}}}\right)+\frac{\omega_{\mathrm{p}, \mathrm{e}}^{2}}{k^{2} v_{\mathrm{th}, \mathrm{e}}^{2}}[1+\psi] \\
\psi=2 \frac{\omega}{k_{\|} v_{\mathrm{th}, \mathrm{e}}}\left(1-\frac{k_{y} V_{\mathrm{d}, \mathrm{e}}}{\omega}\right) \int_{0}^{\infty} d x x \exp \left(-x^{2}\right) J_{0}^{2}\left(k_{\perp} \rho_{\mathrm{e}} x\right) \mathrm{Z}\left(\frac{\omega-k_{y} V_{\mathrm{B}} x^{2}}{k_{\|} v_{\mathrm{th}, \mathrm{e}}}\right)
\end{gathered}
$$

where $Z(x)$ is the plasma dispersion function, $\omega_{\mathrm{pe} / \mathrm{i}}$ is the electron/ion plasma frequency, $V_{\mathrm{d}, \mathrm{e}}$ is the electron diamagnetic drift speed, $V_{\mathrm{B}}=\beta V_{\mathrm{d}, \mathrm{e}} / 2$ is the electron $\nabla B$ drift velocity, $x=v_{\perp} / v_{\text {th,e }}$, and $V$ is the cross-field ion drift. Using measured profiles of $B_{\mathrm{z}}, n_{\mathrm{e}}$, and $T_{\mathrm{e}}$ at $t=264 \mu \mathrm{s}$, the profile of the maximum LHDI growth rate was computed using Eq. 1 (assuming $\left.T_{\mathrm{i}} / T_{\mathrm{e}}=1,2,3\right)$ and is shown in Fig. 3(b). The computed maximum growth rate profile is asymmetric with respect to the current sheet and compares well with the fluctuation amplitude profile at $t=264 \mu \mathrm{s}$. The asymmetry in the predicted growth rate comes from asymmetries in the measured density gradient (which drives the instability) and the magnetic field profile across the current sheet at $t=264 \mu \mathrm{s}$, as shown in Fig. 3(c). These two asymmetries, which are consistent with radial force balance [2], combine to produce a strong asymmetry in the plasma beta across the current sheet, with a larger, stabilizing beta on the outer edge.

The observed decrease in the fluctuation amplitude after $t=264 \mu$ s could be due to ion heating during reconnection, since the linear growth rate of the LHDI decreases with increasing $T_{\mathrm{i}} / T_{\mathrm{e}}$ (see Fig. 3(b)). Spectroscopic ion temperature measurements in Helium discharges in MRX have shown significant ion heating to temperature ratios of $T_{\mathrm{i}} / T_{\mathrm{e}} \lesssim 2$ during reconnection [15]. In hydrogen, direct ion temperature measurements have not been performed, however estimates of $T_{\mathrm{i}}$ based on radial force balance yield temperature ratios 
of $T_{\mathrm{i}} / T_{\mathrm{e}} \gtrsim 3$ during the reconnection process. Ion heating by processes associated with reconnection, perhaps including the LHDI turbulence itself, could thus result in suppression of the LHDI and could explain the rapid drop of the fluctuation amplitude in time.

Spatial correlations in the LHDI turbulence were also studied, using spatially separated floating double Langmuir probes. Measurements were performed using probe spacings of $1,3.5$, and $10 \mathrm{~mm}\left(\rho_{\mathrm{e}} \sim 0.5 \mathrm{~mm}\right)$. Fig. 4(a) shows the squared coherency, averaged over 20 similar discharges per separation, for each of the three probe separations. The average decorrelation length in the turbulence can be estimated from this data to be several $\rho_{\mathrm{e}}$, which is comparable to the wavelength at peak growth for the LHDI $\left(\lambda \sim 2 \pi \rho_{\mathrm{e}}\right)$. This observation is consistent with the predicted strong linear growth rate for the LHDI in MRX. Computation of statistical dispersion relations for the measured fluctuations was done using the phase in the cross-spectrum of $1 \mathrm{~mm}$ separated probe signals [17]. Dispersion relations for probes orientated in the electron $\left(0^{\circ}\right)$ and ion $\left(180^{\circ}\right)$ diamagnetic directions are shown in Fig. 4(b). The waves show a preference for propagation in the electron diamagnetic direction, which is consistent with the LHDI in the ion rest frame. Spectroscopic measurements of average ion flows in the MRX current sheet have indicated that the ions are at rest in the lab frame [15]. A statistically significant determination of the phase velocity of the fluctuations is precluded by the observation of a wide range of phase shifts at each frequency in the turbulence. This is evident in the spectral width of the $k_{\perp}$ measurement, which is represented by the gray regions in the figure. This observation might be explained by nonlinear modifications to the wavenumber spectrum, as the strongly-growing LHDI should quickly saturate nonlinearly.

The final point to address in this Letter is the role of the measured turbulence in the process of magnetic reconnection in MRX. The time behavior of the fluctuations compared to that of the reconnection current and electric field, as shown in Fig. 5, suggest that these fluctuations do not play an essential role in reconnection in MRX. In this figure, the electric field, which is directly related to the reconnection rate, and plasma current seem fairly insensitive to the decay and eventual disappearance of the fluctuations. The measurements reported here are done only near $z=0$, and it is possible that the fluctuations persist 
elsewhere in the current sheet. Even if they were to persist elsewhere, the lack of fluctuations at the center of the current sheet rules out local generation of anomalous resistivity by the LHDI through turbulent scattering of the current carrying particles. An additional set of data was taken to explore the dependence of the fluctuation amplitude on the collisionality in MRX current sheets. If the fluctuations were in some other way responsible for providing anomalous resistivity in MRX current sheets, one might expect that the amplitude would be suppressed at high collisionality, where $E_{\theta} / \eta_{\mathrm{sp}} j_{\theta} \sim 1$. Fig. 6(a) shows the measured peak fluctuation amplitude (peak amplitude in both space and time) versus $\lambda_{\mathrm{mfp}} / \delta$ from a scan of fill pressure. The amplitude of the fluctuations does tend to increase with decreasing collisionality (increasing $\lambda_{\mathrm{mfp}} / \delta$ ). However, if the fluctuation amplitude is normalized to the measured electron temperature, which might be considered a crude estimate of $\delta n / n$ in the turbulence, we find that there is essentially no change in this quantity with collisionality, as shown in Fig. 6(b). The presence of significant fluctuation amplitude in high collisionality discharges, where Spitzer resistivity is sufficient to balance the reconnection electric field, casts further doubt on the role of the LHDI in generating any significant turbulent resistivity in MRX.

In conclusion, we have made detailed measurements of fluctuations in MRX, resulting in the first observation of the lower hybrid drift instability in a laboratory current sheet. The measurements described here suggest that the LHDI is not responsible for providing turbulent resistivity enhancements in MRX current sheets. The diagnostics used in these studies are expected to be capable of detecting fluctuations over a wide range of frequencies $\left(f \lesssim 10 f_{\mathrm{LH}}\right)$, and the fact that the LHDI is currently the only observed fluctuation during the quasi-steady reconnection phase makes it difficult to construct a theoretical picture of reconnection in MRX using turbulent anomalous resistivity. Other much lower frequency magnetic fluctuations are observed in the center of the current sheet near the end $(t \gtrsim 280 \mu \mathrm{s})$ of the quasi-steady reconnection phase in MRX, but discussion of these fluctuations will be reserved for a later paper. The results reported here may also be relevant to reconnection in space plasmas, such as in the magnetotail, where evidence for the presence of the LHDI 
exists.

The authors thank D. Cylinder and R. Cutler for their excellent technical assistance and S. Hsu for useful discussions. TAC acknowledges support from a NASA GSRP Fellowship. MRX is jointly funded by DOE, NASA, and NSF. 


\section{REFERENCES}

[1] D. Biskamp, Phys. Rep. 237, 179 (1994).

[2] M. Yamada et al., Phys. Plas. 7, 1781 (2000).

[3] D. Biskamp, E. Schwarz, and J.F. Drake, Phys. Rev. Lett. 75, 3850 (1995).

[4] E. Parker, Ap. J. 414, 389 (1993).

[5] J. Birn et al., J. Geophys. Res. Space. Phys. 106, 3715 (2001).

[6] R. Davidson et al., Phys. Fluids 20, 301 (1977), and references therein.

[7] J. Huba, N. Gladd, and K. Papadopoulos, Geophys. Res. Lett. 4, 125 (1977).

[8] R. Horiuchi and T. Sato, Phys. Plasmas 6, 4565 (1999).

[9] B. Rogers, J. Drake, and M. Shay, Geophys. Res. Lett. 27, 3157 (2000).

[10] I. Shinohara et al., J. Geophys. Res. 103, 20365 (1998).

[11] P. Yoon, A. Lui, and C. Chang, Phys. Plasmas 1, 3033 (1994).

[12] W. Gekelman and R. Stenzel, J. Geophys. Res. 89, 2715 (1984).

[13] M. Yamada et al., Phys. Plasmas 4, 1936 (1997).

[14] H. Ji et al., Phys. Rev. Lett. 80, 3256 (1998).

[15] S. Hsu et al., Phys. Plasmas 8, 1916 (2001).

[16] J. Huba and C. Wu, Phys. Fluids 19, 988 (1976).

[17] J. Beall, Y. Kim, and E. Powers, J. Appl. Phys. 53, 933 (1982). 


\section{FIGURES}
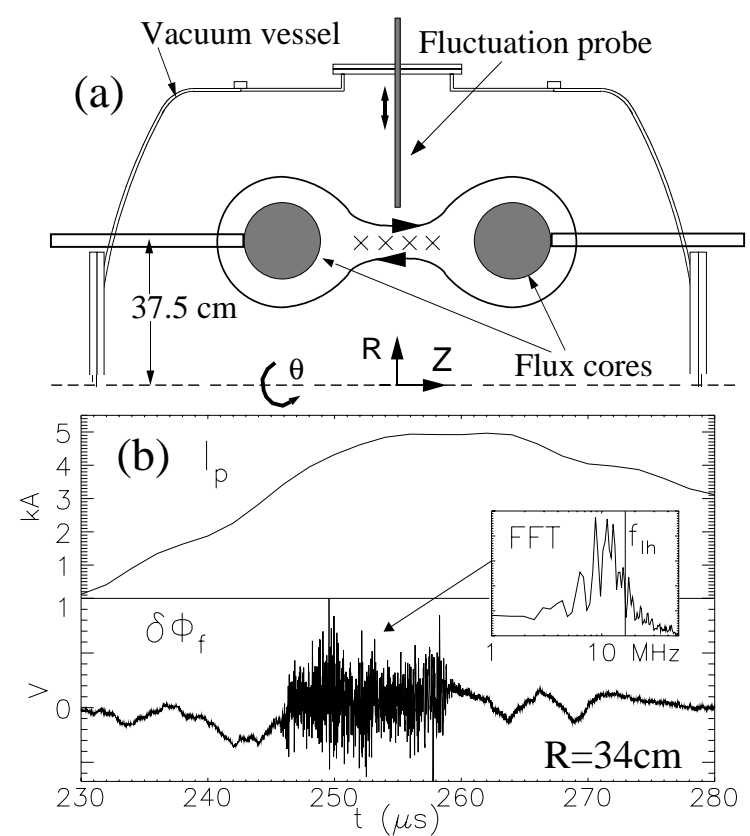

FIG. 1. (a) Schematic of the MRX apparatus with fluctuation probe. Current sheet location is denoted by x's. Probe shaft width $(1 / 4-3 / 8$ ") is not to scale. (b) Traces of plasma current and measured floating potential signal along with an FFT of the signal. Current sheet formation and reconnection occur roughly from $t=240 \mu$ s to $280 \mu \mathrm{s}$.

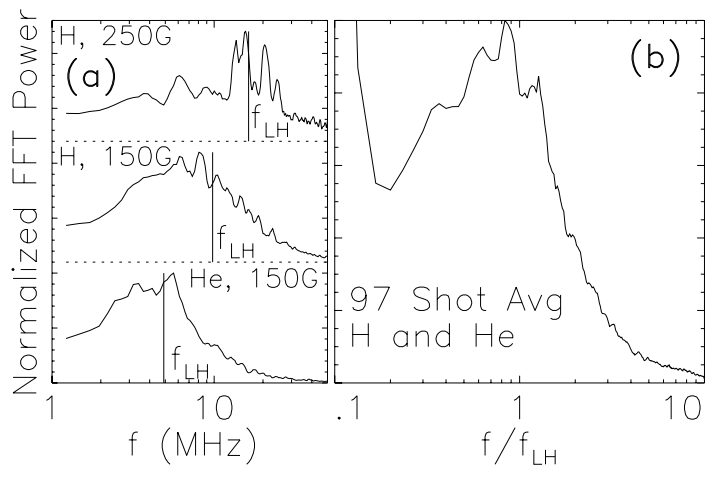

FIG. 2. (a) Average floating potential power spectrum in helium at $150 \mathrm{G}$ and hydrogen at $150 \mathrm{G}$ and 250G. (b) Average power spectrum for 97 discharges, with frequency normalized to the local lower hybrid frequency for each discharge. 


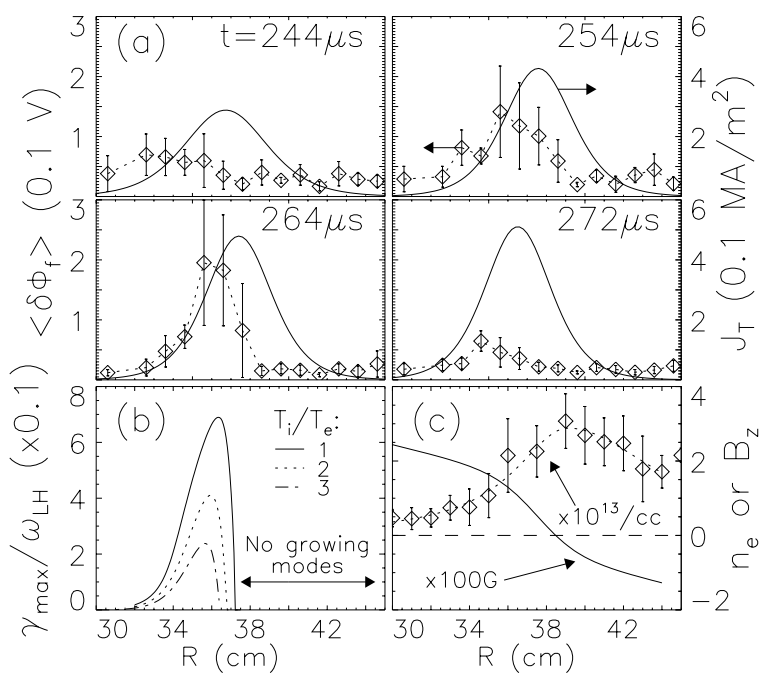

FIG. 3. (a) Average radial profile of root mean square floating potential fluctuation amplitude at four time points. (b) Maximum LHDI linear growth rate profile computed using measured plasma parameter profiles at $t=264 \mu \mathrm{s}$. (c) Profiles of $n_{\mathrm{e}}$ and $B_{\mathrm{z}}$ showing asymmetries which explain the asymmetric fluctuation amplitude profile.
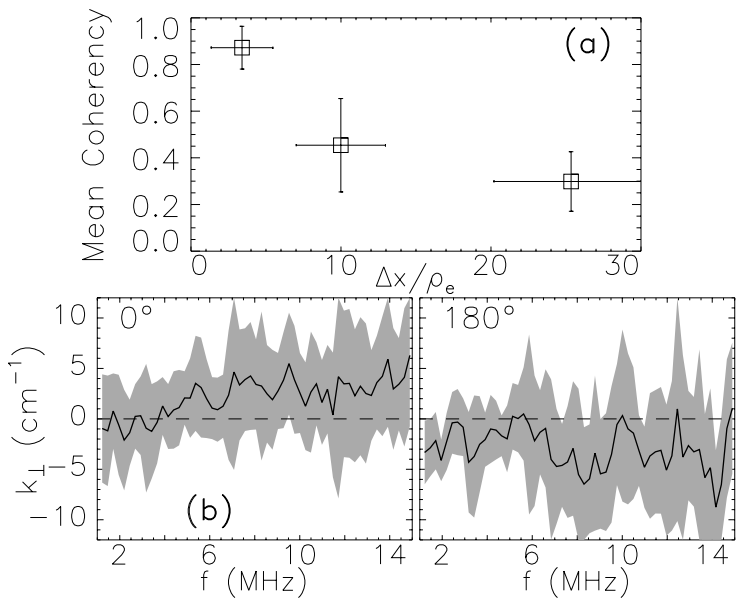

FIG. 4. (a) Mean squared coherency in spatially separated floating potential measurements. (b) Statistical dispersion relations at $1 \mathrm{~mm}$ separation, for two probe orientations $\left(0^{\circ} / 180^{\circ}=\right.$ electron/ion diamagnetic direction). 


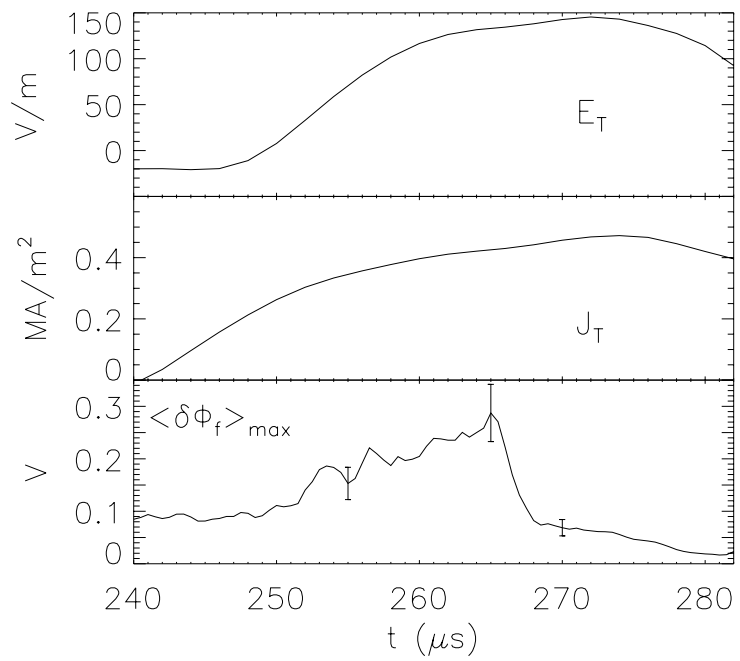

FIG. 5. Time history of toroidal electric field at the null line, peak current density, and peak (in space) fluctuation amplitude.

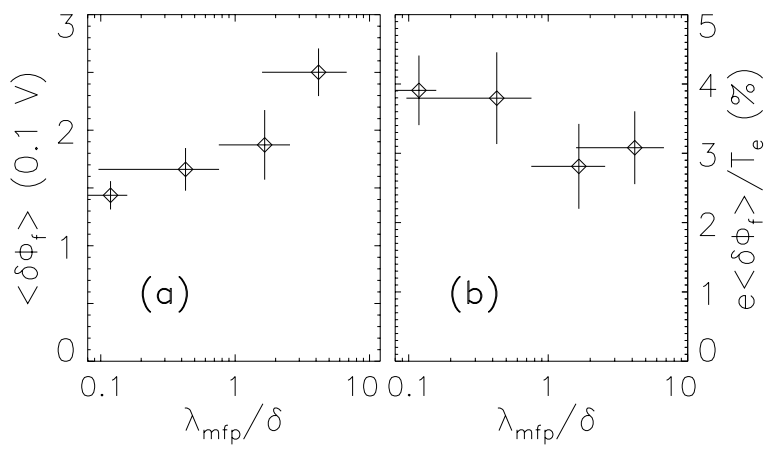

FIG. 6. (a) Fluctuation amplitude and (b) normalized fluctuation amplitude versus collisionality from a scan in fill pressure. 


\section{External Distribution}

Plasma Research Laboratory, Australian National University, Australia

Professor I.R. J ones, Flinders University, Australia

Professor J oão Canalle, Instituto de Fisica DEQ/IF - UERJ , Brazil

Mr. Gerson O. Ludwig, Instituto Nacional de Pesquisas, Brazil

Dr. P.H. Sakanaka, Instituto Fisica, Brazil

The Librarian, Culham Laboratory, England

Library, R61, Rutherford Appleton Laboratory, England

Mrs. S.A. Hutchinson, JET Library, England

Professor M.N. Bussac, Ecole Polytechnique, France

Librarian, Max-Planck-Institut für Plasmaphysik, Germany

J olan Moldvai, Reports Library, MTA KFKI-ATKI, Hungary

Dr. P. Kaw, Institute for Plasma Research, India

Ms. P.J . Pathak, Librarian, Insitute for Plasma Research, India

Ms. Clelia De Palo, Associazione EURATOM-ENEA, I taly

Dr. G. Grosso, Instituto di Fisica del Plasma, Italy

Librarian, Naka Fusion Research Establishment, J AERI, J apan

Library, Plasma Physics Laboratory, Kyoto University, J apan

Research Information Center, National Institute for Fusion Science, J apan

Dr. O. Mitarai, Kyushu Tokai University, J apan

Library, Academia Sinica, Institute of Plasma Physics, People's Republic of China

Shih-Tung Tsai, Institute of Physics, Chinese Academy of Sciences, People's Republic of China

Dr. S. Mirnov, TRINITI, Troitsk, Russian Federation, Russia

Dr. V.S. Strelkov, Kurchatov Institute, Russian Federation, Russia

Professor Peter Lukac, Katedra Fyziky Plazmy MFF UK, Mlynska dolina F-2, Komenskeho Univerzita, SK-842 15 Bratislava, Slovakia

Dr. G.S. Lee, Korea Basic Science Institute, South Korea

Mr. Dennis Bruggink, Fusion Library, University of Wisconsin, USA

Institute for Plasma Research, University of Maryland, USA

Librarian, Fusion Energy Division, Oak Ridge National Laboratory, USA

Librarian, Institute of Fusion Studies, University of Texas, USA

Librarian, Magnetic Fusion Program, Lawrence Livermore National Laboratory, USA

Library, General Atomics, USA

Plasma Physics Group, Fusion Energy Research Program, University of California at San Diego, USA

Plasma Physics Library, Columbia University, USA

Alkesh Punjabi, Center for Fusion Research and Training, Hampton University, USA

Dr. W.M. Stacey, Fusion Research Center, Georgia Institute of Technology, USA

Dr. J ohn Willis, U.S. Department of Energy, Office of Fusion Energy Sciences, USA

Mr. Paul H. Wright, Indianapolis, Indiana, USA 
The Princeton Plasma Physics Laboratory is operated by Princeton University under contract with the U.S. Department of Energy.

\author{
Information Services \\ Princeton Plasma Physics Laboratory \\ P.O. Box 451 \\ Princeton, NJ 08543
}

Phone: 609-243-2750

Fax: 609-243-2751

e-mail: pppl_info@pppl.gov

Internet Address: http://www.pppl.gov 